\title{
Necrotising Otitis Externa: Can treatment be de-escalated?
}

\author{
Michael Mather ${ }^{1}$, Arpita Singh ${ }^{2}$, and Puveendran Arullendran ${ }^{2}$ \\ ${ }^{1}$ Newcastle University Institute of Cellular Medicine \\ ${ }^{2}$ Sunderland Royal Hospital
}

June 22, 2020

\begin{abstract}
Introduction: Necrotising otitis externa (NOE) is a severe infection of the external auditory canal and the incidence in the UK has risen dramatically in recent years. Treatment options for NOE remain contentious and can confer substantial risks. Osteomyelitis at other anatomical sites has shown promising outcomes when treated with early debridement and short-course antimicrobial therapy. Application of this paradigm in NOE may offer distinct advantages for patients but this remains to be demonstrated. Methods: A prospective cohort analysis of patients with NOE admitted to Sunderland Royal Hospital between January-September 2019. Previous treatment, examination findings, medical history, relevant microbiology and imaging results were document. Results: A total of eight patients were included (age 58-87, male:female 6:2). Most (6/8) patients had type 2 diabetes; $5 / 6$ had poor glycaemic controls (HbA1c $>48 \mathrm{mmol} / \mathrm{mol}$ ). 4/8 patients had undergone previous ear syringing. Pain was present on average for 40 days prior to admission (range 14-60 days). Otoscopy and CT findings were highly variable between cases. Most (5/8) patients grew P. aeruginosa on bacterial swabs. 5/8 patients were treated with short course antimicrobial therapy (3/8 prolonged). 5/8 received early surgical debridement. $7 / 8$ patients were successfully treated -1 patient died of concurrent haematological malignancy. Conclusion: A standardised protocol for NOE has facilitated a consistent approach to treatment at our centre. Selected cases have good outcomes with short course antimicrobial therapy and early surgical debridement, but this analysis was not powered to determine statistical significance. A randomised trial with more patients would confirm non-inferiority of short course antimicrobial therapy and early surgical debridement in NOE.
\end{abstract}

\section{Five succinct key points:}

1. Patients with NOE often have medical comorbidities which require concurrent optimization to improve outcomes.

2. A novel grading system of otoscopy and CT scan findings presented herein provides an objective measure and common language with which to describe extent of NOE.

3. Initial outcomes data suggest selected patients make a good recovery with short course intravenous antimicrobial therapy as compared to those on long course intravenous antimicrobial therapy but an appropriately powered randomized trial is needed to confirm non-inferiority.

4. Limited surgical debridement provides an opportunity for histological and microbiological testing in cases refractory to initial management or persistent physical signs.

5. Multidisciplinary involvement - in particular with microbiology and radiology is essential to optimize management.

\section{INTRODUCTION}

Necrotising otitis externa (NOE) is a severe infection of the external auditory canal which can progress to skull base osteomyelitis, cranial nerve palsies, meningitis, and is frequently fatal (1). The incidence of NOE in the UK has risen almost $600 \%$ between 2002-2017 - which may be due to a concomitant rise in relevant 
risk factors such as advancing age and diabetes mellitus (2)(3). Treatment frequently involves prolonged treatment with parenteral antimicrobial therapy, which often necessitates an extended hospital admission and its associated risks, including nosocomial infection.

The extent of disease in NOE is variable and management remains contentious. Promise has been shown in other forms of osteomyelitis for early debridement and short course antimicrobial therapy (4). Although the application of this paradigm to NOE offers several advantages for patient care, the potential of de-escalation of treatment in selected cases of NOE remains to be demonstrated.

\section{METHODS}

The study comprises a prospective cohort analysis comparing short course antimicrobial therapy +/- early surgical debridement versus prolonged course antimicrobial therapy alone in patients with NOE. The treatment protocol was approval by the Head \& Neck Clinical Governance team at South Tyneside and Sunderland NHS Foundation Trust. All patients admitted with a clinical diagnosis of NOE to Sunderland Royal Hospital (SRH) between January-September 2019 were included.

Factors evaluated on admission included duration of symptoms, any relevant microbiology results, antibiotic therapy to date, history of ear syringing, patient pain score (1-10), cranial nerve palsies, and medical comorbidities (e.g. diabetes mellitus).Baseline investigations, including an ear swab, baselines blood tests (FBC, U\&E, LFT, glucose, HbA1c, CRP, ESR), and a CT temporal bone, were performed for each patient.

In all cases the ear was examined by a senior member of the ENT team (registrar or consultant) and the canal was given a staging score (see Fig. 1):

Stage $\mathbf{1}=$ Normal or near normal ear canal.

Stage $\mathbf{2}=$ All of tympanic membrane visible, abnormal ear canal with granulation, polyp or swelling.

Stage $\mathbf{3}=$ more than $50 \%$ of tympanic membrane visible, due to polyp, granulation or swollen ear canal.

Stage $4=$ less than $50 \%$ of tympanic membrane visible, due to polyp, granulation or swollen ear canal.

A significant improvement was defined as an improvement of 2 stages (i.e. stage 4 to stage 2, or stage 3 to stage 1).

A novel CT grading system was proposed to grade the degree of osteomyelitis (see Fig. 2):

Stage $\mathbf{1}=$ Soft tissue swelling, no osteomyelitis.

Stage $\mathbf{2}=$ Localised osteomyelitis to ear canal

Stage 3 = Evidence of inflammatory disease extending beyond ear canal, but not extending out of temporal bone.

Stage 4 = Disease extending beyond temporal bone OR presence of cranial nerve abnormalities.

If there were cranial nerve deficits, or the CT scan was stage $3 / 4$, or the diagnosis was unclear, then an MRI scan was requested to evaluate soft tissue extent and meningeal involvement. Where the duration of antibiotics was to be extended beyond 6 weeks a gallium and bone scan was undertaken to extent of evaluate resolution and monitor treatment.

Patients were routinely commenced on intravenous piperacillin-tazobactam and gentamicin in combination. Review by a single surgeon (PA), based upon a database of previous NOE outcomes, determined whether patients were then enrolled on short course (2 weeks) +/- early debridement versus long course (6+ weeks) intravenous antimicrobial therapy.

Review of historical data (unpublished) suggested that the following parameters conferred a good prognosis:

- Absence of comorbidities

- Short duration of preceding symptoms 
- Resolution of pain score down to patient reported score of $0 / 10$ within 4 days

- Moving down 2 steps on otoscopy grading scale within 1 week

- CT findings limited to grade 1 or 2

Patients who met these criteria were enrolled on 2 weeks of intravenous (short course) antimicrobial therapy, and were subsequently switched to oral ciprofloxacin for a further 4-week course of treatment. All other patients were assigned to long course (6 weeks intravenous) antimicrobial therapy either had their entire treatment as an inpatient or administration in the community via a long line inserted prior to discharge.

Surgical intervention was considered only in selected circumstances; specifically:

A) Presence of an ear canal lesion that fails to respond to treatment after 1 week. In this case a biopsy with or without debridement wasoffered.

B) If at the end of treatment there was an area of necrotic bone that had not epithelised, or a bony sequestrum was present, then surgical removal wasoffered.

C) Early in course of disease for pain relief where this failed with improve with medical therapy alone.

\section{RESULTS}

\section{Demographics}

8 patients were included. Age range 58-87 years with male:female ratio of 6:2. The majority of patients (6/8) had type 2 diabetes; and $5 / 6$ had poor diabetes control (HbA1c $>48 \mathrm{mmol} / \mathrm{mol}) .4 / 8$ patients had underwent prior ear syringing ( $3 / 8$ had not, and $1 / 8$ was unsure).

\section{AssessmentE Treatment}

Patients had pain on average for 40 days prior to admission (range 14-60 days). Otoscopy and CT scan grading was highly variable between cases (Table 1).6/8 patients had had an ear swab taken prior to admission. $5 / 8$ patients grew $P$. aeruginosa and 1/8 grew S. lugdunensis . 2/8 demonstrated no bacterial growth. 1/8 demonstrated resistance to gentamicin. Patient treatment and outcomes are summarised in Table 2.

\section{DISCUSSION}

NOE presents a challenge both in terms of diagnosis and treatment. It is an uncommon condition which makes large studies difficult to conduct and, as such, management options remain poorly defined. Most clinicians agree that intravenous antibiotics and meticulous glycaemic control are essential, but the role of surgical intervention, amongst other factors, remains controversial. Indeed, a survey of over 200 UK otolaryngologists reveals surprising disparities in management (5).

Evidence from orthopaedic literature concerning osteomyelitis at subsites other than the external auditory canal has suggested that short courses of antimicrobial therapy may be suitable for particular patient groups (4). Short course may offer distinct advantages over long course antimicrobial therapy; such as fewer drug side effects, lower financial cost, shorter length of hospital admission, and is more convenient for patients. There are also advantages to limited surgical debridement, including de-bulking microbial load, facilitating microbiological testing, and permit histological analysis for exclusion of malignancy. Indeed other groups have suggested that deep tissue cultures may be more useful than superficial swabs (6).

To our knowledge this has not been investigated in NOE until the present study. In this series allocation to short course +/- surgery vs long course antimicrobial therapy was determined based on a historical database of outcomes in NOE treated with a standard long course of antimicrobial therapy at SRH. We previously identified (unpublished) positive prognostic factors, and where these are present the patients were allocated to short course antimicrobial therapy $+/$ - surgery (see methods). All other patients were allocated to long course antimicrobial therapy.

Most patients in this series were elderly and most were male. The majority had extensive comorbidities as measured by the Charleston comorbidity index; in particular diabetes mellitus, which was often poorly 
controlled; predisposing them to infection. Interestingly, despite the classical association with NOE, recent data suggests that diabetes mellitus is not an independent risk factor for 30-day readmission, prolonged length of stay, or discharge to a rehabilitation facility (7). Many patients in this series grew bacteria typical for NOE, such as P. Aeruginosa (8). Few demonstrated no bacterial growth, which may be a function of having received treatment prior to microbiological analysis.

Most patients in this series presented with advanced disease, as measured by otoscopy and CT findings. To be able to compare findings between patients it is important to have a scoring system to objectively describe extent of disease. We therefore developed a simple and intuitive distinct grading system for each, as outlined in methods, which offer a common language to clinicians that is currently missing in NOE practice.

Although most patients receiving short course antimicrobial therapy +/- surgery had positive outcomes, this series is small and not statistically powered to definitely compare treatment arms. Importantly, however, we identified that all but one patient in this series survived and had good outcomes. The patient who did not survive died of related comorbidities, rather than from complications of NOE. This suggests a larger trial comparing short course antimicrobial therapy $+/$ - surgery versus long course antimicrobial therapy for non-inferiority would be feasible and may well offer distinct advantages for selected patients.

\section{CONCLUSION}

Our experience of using a standardized protocol for investigating and treating patients with NOE helped to facilitate a consistent approach within the department. We found selected patients obtain good outcomes with short course intravenous antimicrobial therapy $+/$ - early debridement but this study was not powered to determine statistical significance. A randomised trial with more patients would confirm evidence of non-inferiority of short course treatment in NOE.

Table 1: Otoscopy and CT scan grading on admission

\begin{tabular}{lll}
\hline Patient number & Otoscopy grade & CT scan grade \\
\hline $\mathbf{1}$ & 1 & 3 \\
$\mathbf{2}$ & 4 & 2 \\
$\mathbf{3}$ & 4 & 3 \\
$\mathbf{4}$ & 4 & 3 \\
$\mathbf{5}$ & 2 & 1 \\
$\mathbf{6}$ & 4 & 1 \\
$\mathbf{7}$ & 1 & 3 \\
$\mathbf{8}$ & 2 & 1 \\
\hline
\end{tabular}

Table 2: Patient treatment and outcomes.

\begin{tabular}{lll}
\hline & Treatment & Number of patients \\
\hline Prior to hospital admission & Topical antibiotics & $3 / 8$ \\
& Oral antibiotics & $1 / 8$ \\
Length of intravenous antimicrobial therapy* & Short course & $5 / 8$ \\
& Long course & $3 / 8$ \\
Surgical intervention & Conservative & $3 / 8$ \\
& Debridement & $5 / 8$ \\
Outcomes** & Survived & $7 / 8$ \\
& Died & $1 / 8$ \\
\hline
\end{tabular}

*2 complex patients had 6 weeks intravenous antimicrobial therapy ( 1 clival involvement, 1 cranial nerve 
palsy). 1 patient received 2 weeks intravenous antimicrobial therapy and self-discharged.

** One patient died from concurrent haematological malignancy.

\section{REFERENCES:}

1. Chandler JR. Malignant external otitis. Laryngoscope [Internet]. 1968 Aug [cited 2020 Apr 12];78(8):1257-94. Available from: http://www.ncbi.nlm.nih.gov/pubmed/4970362

2. Hospital Episode Statistics (HES) - NHS Digital [Internet]. [cited 2019 Oct 23]. Available from: https://digital.nhs.uk/data-and-information/data-tools-and-services/data-services/hospital-episodestatistics

3. Hutson KH, Watson GJ. Malignant otitis externa, an increasing burden in the twenty-first century: review of cases in a UK teaching hospital, with a proposed algorithm for diagnosis and management. J Laryngol Otol [Internet]. 2019 May [cited 2020 Apr 12];133(5):356-62. Available from: http://www.ncbi.nlm.nih.gov/pubmed/30975233

4. Huang C-Y, Hsieh RW, Yen H-T, Hsu T-C, Chen C-Y, Chen Y-C, et al. Short- versus long-course antibiotics in osteomyelitis: A systematic review and meta-analysis. Int J Antimicrob Agents [Internet]. 2019 Mar [cited 2020 Apr 12];53(3):246-60. Available from: http://www.ncbi.nlm.nih.gov/pubmed/30639627

5. G C, M P, S D, I B. Current Management of Necrotising Otitis Externa in the UK: Survey of 221 UK Otolaryngologists. Acta Otolaryngol [Internet]. 2017 [cited 2020 Apr 12];137(8). Available from: https://pubmed.ncbi.nlm.nih.gov/28301961/

6. Peled C, Kraus M, Kaplan D. Diagnosis and treatment of necrotising otitis externa and diabetic foot osteomyelitis - similarities and differences. J Laryngol Otol [Internet]. 2018 Sep [cited 2020 Apr 12];132(9):7759. Available from: http://www.ncbi.nlm.nih.gov/pubmed/30149824

7. Schwam ZG, Ferrandino R, Kaul VZ, Wanna GB, Cosetti MK. Thirty-Day Readmission and Prolonged Length of Stay in Malignant Otitis Externa. Laryngoscope [Internet]. 2019 Nov 22 [cited 2020 Apr 12]; Available from: http://www.ncbi.nlm.nih.gov/pubmed/31758583

8. MJ C, BW K. Malignant Otitis Externa. Otolaryngol Clin North Am [Internet]. 2008 [cited 2020 Apr 12];41(3). Available from: https://pubmed.ncbi.nlm.nih.gov/18435997/

The data that support the findings of this study are available from the corresponding author upon reasonable request.

This study received no specific funding.

\section{Hosted file}

image1. emf available at https://authorea.com/users/335661/articles/461496-necrotising-otitisexterna-can-treatment-be-de-escalated

Figure 1: Canal staging system

\section{Hosted file}

image2. emf available at https://authorea.com/users/335661/articles/461496-necrotising-otitisexterna-can-treatment-be-de-escalated

Figure 2: Grading system of extent of osteomyelitis based upon CT imaging 